\title{
Cascade control algorithm of test bench for studying load- sensing electrohydraulic steering systems
}

\author{
Alexander Mitov ${ }^{1, *}$, Jordan $\mathrm{Kralev}^{2}$, and Ilcho Angelov ${ }^{1}$ \\ ${ }^{1}$ Technical University - Sofia, Department of Hydroaerodynamics \& Hydraulic machines, Bulgaria \\ ${ }^{2}$ Technical University - Sofia, Department of Systems \& Control, Bulgaria
}

\begin{abstract}
The paper presents the design and synthesis of a three-loop cascade control system with a digital PI controller intended for installation in a test bench for load-sensing electrohydraulic steering units. The structure and layout of both the hydraulic system and the control system are shown. The cascade control system consists of three controllers for measurable variables: proportional spool valve position, steering velocity and steering cylinder position. The developed control algorithm is embedded into a 32-bit microcontroller for mobile applications. Experimental studies have been carried out to present the performance of the entire electrohydraulic system. The developed control algorithm will serve as a basis for further study of the system with advanced control techniques implementable in low speed mobile machines.
\end{abstract}

\section{Introduction}

The need for mobile machines with automated control of the direction of movement is a determining factor for the development of built-in electrohydraulic steering systems. A basic component in these systems is the electrohydraulic steering unit (EHSU). Modern EHSUs enable the reconciliation of two modes of steering depending on the control action: mechanical - through the steering wheel and digital - by electronic joystick or GPS signal. In this way, besides meeting the requirements of the safety standards, new advantages are gained with regard to precise remote control and provision of variable steering ratio between the steering wheel and the steered wheels [1].

In addition, the implementation of modern loadsensing (LS) steering systems on the executive servo cylinder significantly influences the energy balance of the entire hydraulic drive system, providing the ability to adjust the input hydraulic power by two parameters - flow rate and pressure. This adjustment capability combined with the relatively high efficiency of individual displacement volume machines results in a high structural efficiency of the entire hydraulic system [2].

It should be noted that in this type of steering systems there is no mechanical connection between the steering wheel and the steering axle of the machine, such as the conventional steering system of cars [3]. The transmission of energy is carried out by means of a hydraulic working fluid. This makes them applicable to mobile machines whose speed does not exceed $60 \mathrm{~km} / \mathrm{h}$ [4].

On the other hand, in order to meet the requirements for the compactness of the steering devices, related mainly to the limitations of the construction of the mobile machinery, the conversion of the electrical signal into a hydraulic flow is achieved through a digital electrohydraulic control module (EHCM). The continuous development of this type of modules is an example for one of the applications of the so called digital fluid power. An example of this is the well-known type of Danfoss - PVE [1]. It has an electrohydraulic system consisting of four micro valves, connected in parallel, which serve for pilot hydraulic control of a proportional spool valve (with position feedback) integrated in the steering unit. The proportional hydraulic control valve determines the direction of movement of the servo cylinder which steers the wheels. The parallel connected valves in the module are switched by solenoids, working in pairs according to pulse width modulation (PWM) and the electric control signal is generated by a microcontroller (Fig.1). The embedded control system has to ensure the quality of the entire electrohydraulic system [5].

The main objective of the paper is to present a developed three-loop cascade system for automatic control of a load-sensing electro-hydraulic steering system that is implementable in a low speed mobile machinery. The hydraulic steering system is evaluated on a developed test bench of the EHSU. The control algorithm uses a classic PI law that is embedded in a 32-bit microcontroller for mobile applications. Experimentally obtained dynamic characteristics in the form of transients processes during a reference tracking confirm the performance of the entire electrohydraulic system.

\section{Laboratory test bench layout}

A hydraulic diagram of a rig for testing the hydraulic system for the load-sensing steering system, shown in Fig. 1, has been developed.

\footnotetext{
*orresponding author: a_mitov@tu-sofia.bg
} 


\subsection{Hydraulic system}

A hydraulic drive system is composed of: a tank (1), a variable displacement axial-piston pump type A10VSO (3), displacement volume $18 \mathrm{~cm}^{3}$ with pressure controller type DR (4), an electrical motor (2), a main pressure-relief valve (6), a pressure filter (9), a gear type flow rate meter (10), a hydrostatic steering device (11), a hydraulic control block (12) and EHCM (13) type PVE. The plant under control is a double-acting symmetrically servo cylinder (22).

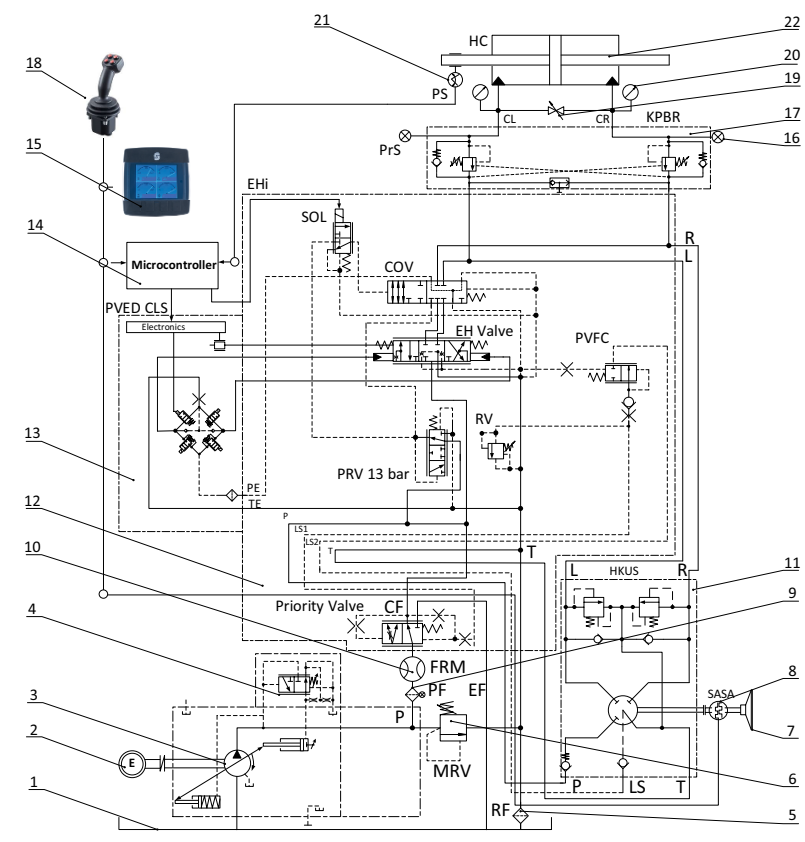

Fig. 1. Internal structure of the target EHSU.

Fig. 2 shows the hydraulic diagram of the pressure controller of the axial-piston pump [6].

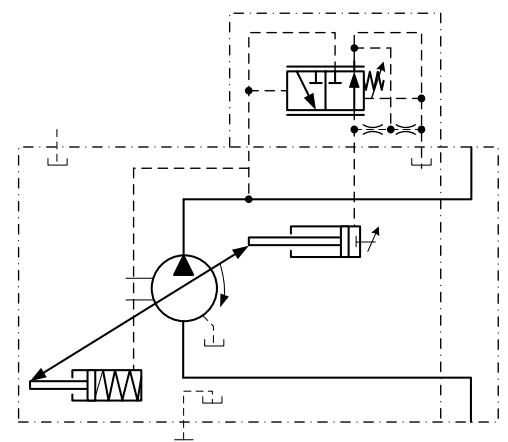

Fig. 2. Hydraulic diagram of a pressure controller - type "DR".

The inclusion of a variable displacement axial-piston pump in the open-loop hydraulic systems makes it possible to adjust the flow rate delivery to the steering system by changing the pump displacement volume from a maximum to a minimum level. The pump is equipped with a DR type pressure control which allows for the reduction of the pump displacement volume, and hence of the supplied flow when the pressure increases (up to the maximum limitation) in the steering system due to the increase in the external force acting on the piston of the servo cylinder.
The developed schematic solution of the test bench complies with the requirement for operation in two modes: mechanical by steering wheel (7) and digital by electronic joystick (18).

The laboratory bench meets also the testing requirements for electrohydraulic steering devices under varying loads. The pressure load is realized by a designed hydraulic block with adjustable over-center valves (17) which are connected to the two chambers of the hydraulic cylinder (22).

\subsection{Control system}

The purpose of the system is to control the stroke of the piston of the servo cylinder (22) by receiving a signal from an electronic joystick (18). The embedded in microcontroller (14) cascade controller calculates a control voltage for an electrohydraulic control module (EHUM, 13) that switches the hydraulically proportional EH valve. The proportional valve determines the direction of the movement of the piston of the servo cylinder (22), supplying working fluid to one of the two chambers (CL and CR). EHCM (13) is constructed of small two-way two-position valves connected in parallel switch by PWM signal.

The developed bench provides the ability to record the experimental dynamic processes, such as starting and stopping the cylinder piston, and monitoring a certain reference (driving law) formed by turning the steering wheel, electronic joystick or pre-programmed into the microcontroller. For this purpose, the rig is equipped with a potentiometric measuring transducer (21) sensing the steering cylinder position.

In addition, pressure sensors (16) are included for measuring the dynamic pressure variation in the two chambers of the cylinder, and in order to allow for operator observation of the values, they are duplicated with high-accuracy pressure gauges (20).

The inclusion of a gear flowmeter (10) allows for a dynamic record of the flow rate supplied from the pump to the hydraulic control block (12).

In the implementations of modern steering systems with architecture according to ISO13849-1, it is necessary to incorporate a sensor (8) for recording the angular position of the steering wheel, respectively [7].

Fig. 3 shows an electrohydraulic steering system model for the purpose of control.

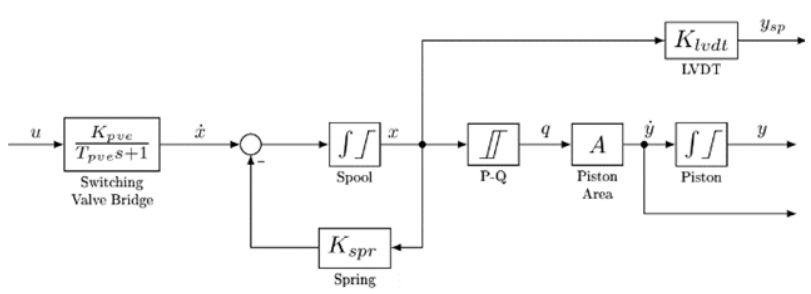

Fig. 3. Open loop interconnection.

The system is designed with dominating linear dynamics, however, some nonlinearities arise - due to naturally nonlinear pressure-flow characteristics and artificially introduced hysteresis for safety reasons. The control signal (manipulated variable) $u$ is a generated 
voltage from the microcontroller [8]. It is converted to a hydraulic signal with the help of a switching valve module (PVE) which can be modelled as a first order dynamical system with gain $K_{p v e}$ and time constant $T_{p v e}$. Switching valves generate a flow, which proportionally moves a directional spool with a velocity $\dot{x}$ such that

$$
\ddot{x}(t)=-\frac{1}{T_{p v e}}\left(-\dot{x}(t)+K_{p v e} u(t)\right) .
$$

The motion of the directional spool is constrained within the range of $\pm 3.5 \mathrm{~mm}$. The spool is returned to a neutral position with the help of equivalent to a springlike action $K_{s p r}$ accounting for actual mechanical spring and hydraulic reaction force arising from jet flow through the spool valve. The position of the directional spool is sensed by a LVDT (linear variable differential transducer) sensor with a gain $K_{l v d t}$ giving a voltage signal $y_{s p}$ to the microcontroller within a range $\pm 2500 \mathrm{mV}$.

Every left or right shift of the spool causes a flow $q$ to the chambers of the steering cylinder, which proportionally to its piston area $A$ translated it in a corresponding direction. Usually, there is a certain deadband in flow response due to the presence of a positive covering in the directional spool valve to protect the device from unintended leaks which can lead to steering drifts. The velocity of the steering cylinder $A$ is integrated to give the piston position measured by a resistive sensor. So the plant has one input port - $u$ and three output ports $-\vec{y}=\left(y_{s p}, y, \dot{y}\right)^{T}$. Since the transfer function from $u$ to $y_{s p}$ is

$$
y_{s p}=\frac{K_{p v e}}{\left(s+K_{s p r}\right)\left(T_{p v e} s+1\right)} u
$$

the dynamics of the direction spool will be dependent on elastic components in the systems accounted in $K_{s p r}$ and pulse-width modulation period related to $T_{p v e}$. The system is constructed so that $T_{p v e} K_{s p r}>10$ and the dominating pole will be $-K_{s p r}$ determining the transient response. The subordinated pole will be $-1 / T_{p v e}$, which will cause some high frequency oscillations around the current spool position due to the switching nature of the PVE. With increasing mechanical spring stiffness and increasing the pressure in the system parameter $K_{s p r}$ will rise, which will make the response faster. Hence, smaller amount of control power will be required to compensate the spool dynamics. Also when decreasing PWM period the time constant $T_{p v e}$ will decrease the amplitude of the steady state oscillations but will increase their frequency. Maximal frequency of the spool oscillations is limited by the mechanical parameters of the switching valves, mainly the mass and friction. Lighter valves will allow faster switching transient and increased frequency.

\section{Design of cascade control algorithm}

Fig. 4 shows the designed cascade control system structure.

It is easy to note the dependability of the plant outputs since a change in the directional spool position will cause a change in steering cylinder velocity which in turn will cause a change in steering cylinder position. Hence, the cascade control technique to achieve steering position tracking is a natural choice. Three regulators are designed: $K_{s p}$ (for spool position), $K_{v}$ (for steering velocity) and $K_{y}$ (for steering cylinder position). The output of an external regulator is seen as a reference signal for the next regulator.

\subsection{Spool position regulator}

The requirements for the spool position controller $K_{s p}$ are for fast rising time and short transients in $y_{s p}$. The steady state error and tracking error are of secondary importance for this control loop. It also has a linearizing effect on the plant, compensating to some extend the dead-band. The controller is implemented as a pure $P I$ with dominating $P$ component [5] $\left(K_{p, s p}>K_{i, s p}\right)$

$$
K_{s p}=K_{p, s p}+K_{i, s p} \frac{1}{s}=K_{p, s p} \frac{s+K_{i, s p} / K_{p, s p}}{s},
$$

where $K_{p, s p}$ is the proportional gain and $K_{i, s p}$ is the integral gain. As a transfer function this regulator has one zero at $-\frac{K_{i, s p}}{K_{p, s p}}$ which can be used to compensate for the dominating pole of the plant at $-K_{s p r}$. So first the proportional gain is increased until the control signal reaches its constraint. Next the integral gain is tuned to compensate the dominating pole and minimize the overshot. The integral gain should not be chosen too high due to possible wind up effects. In the time domain the regulator works at

$$
u\left(k T_{S}\right)=K_{p, s p} e_{s p}\left(k T_{S}\right)+K_{i, s p} x_{i, s p}\left(k T_{S}\right),
$$

where $e_{s p}\left(k T_{S}\right)=r_{s p}\left(k T_{S}\right)-y_{s p}\left(k T_{S}\right)$ is the spool position error calculated every sample $k$ with sample period $T_{S}=0.1 s$. The state of the integrator $x_{i, s p}\left(k T_{S}\right)$ is approximated by the backward Euler difference

$$
x_{i, s p}\left(k T_{S}\right)=x_{i, s p}\left((k-1) T_{S}\right)+T_{S} e_{s p}\left(k T_{S}\right) .
$$

To limit the wind up effect in the integrator its output is limited to a certain percentage of the control range such as $\left|K_{i, s p} x_{i, s p}\left(k T_{S}\right)\right|<\gamma U_{\max }$, where $U_{\max }=5000 \mathrm{mV}$ is the maximal amplitude of the control signal $u$.

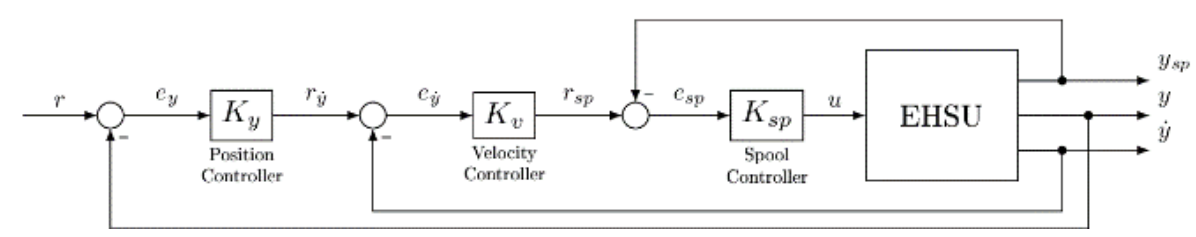

Fig. 4. Cascade control system interconnection. 


\subsection{Steering velocity regulator}

The second control loop takes the translational velocity $\dot{y}$ of the steering cylinder as an input and gives the reference for the spool regulator as its control action $r_{s p}$. The velocity $\dot{y}$ is estimated by calculating the first difference of the measured position signal $y$ by the resistive sensor

$$
\dot{y}_{\text {est }}\left(k T_{S}\right)=\frac{y\left(k T_{S}\right)-y\left((k-1) T_{S}\right)}{T_{S}}
$$

Regulator $K_{v}$ has to react fast to changes in its reference signal $r_{\dot{y}}$ in order to start the steering process as soon as the operator demands it. Such a regulator introduces a derivative like action in the closed loop steering control however minimizing the amplification of the noise in the control signal. Regulator $K_{v}$ is implemented as a high gain $P$ regulator with dead band compensation

$$
r_{s p}\left(k T_{S}\right)=K_{p, v} e_{\dot{y}}\left(k T_{S}\right)+\eta\left(e_{\dot{y}}\left(k T_{S}\right)\right),
$$

where $e_{\dot{y}}\left(k T_{S}\right)=r_{\dot{y}}\left(k T_{S}\right)-\dot{y}\left(k T_{S}\right)$ is the loop error signal and $\eta(e)$ is a compensation term calculated on the basis of dead band range $\left[-\eta_{2}, \eta_{1}\right]$ as

$$
\eta(e)=\left\{\begin{array}{c}
\eta_{1}, e>0 \\
0, e=0 \\
-\eta_{2}, e<0
\end{array} .\right.
$$

\subsection{Steering cylinder position}

The outer regulator $K_{y}$ is designed to achieve the required trajectory tracking performance in the presence of variable load disturbances. Since accuracy is the main requirement this regulator is implemented as a PI regulator with increased integral action while keeping the stability margin

$$
r_{\dot{y}}\left(k T_{S}\right)=K_{p, y} e_{y}\left(k T_{S}\right)+K_{i, y} x_{i, y}\left(k T_{S}\right),
$$

where $e_{y}\left(k T_{S}\right)=r\left(k T_{S}\right)-y\left(k T_{S}\right)$ is the error between the desired piston position and the one measured by the resistive sensor. The integrator term $x_{i, y}$ is implemented as a backward Euler difference with included backtracking anti-wind up term $w\left(k T_{S}\right)$

$$
x_{y, s p}\left(k T_{S}\right)=x_{i, s p}\left((k-1) T_{S}\right)+T_{S} e_{s p}\left(k T_{S}\right)+T_{S} K_{t} w\left(k T_{S}\right) .
$$

The amount of anti-wind up action is tuned with the parameter $K_{t}$. If it is higher the overshot will be attenuated but if it is too high the integrator will not work effectively. The $w\left(k T_{S}\right)$ term is calculated as

$$
w\left(k T_{S}\right)=u_{\text {sat }}\left(k T_{S}\right)-u_{\text {unsat }}\left(k T_{S}\right),
$$

where $u_{\text {sat }}$ is the saturated control action and $u_{\text {unsat }}$ is the unsaturated control action.

\section{Experimental results}

Reference tracking experiments with typical for steering applications trajectory are used to confirm the prescribed closed-loop performance. They are presented in Figures 5 to 7 when the reference signal is generated with a joystick and in Figures 8 to 10 when the reference signal is preprogrammed in the microcontroller.

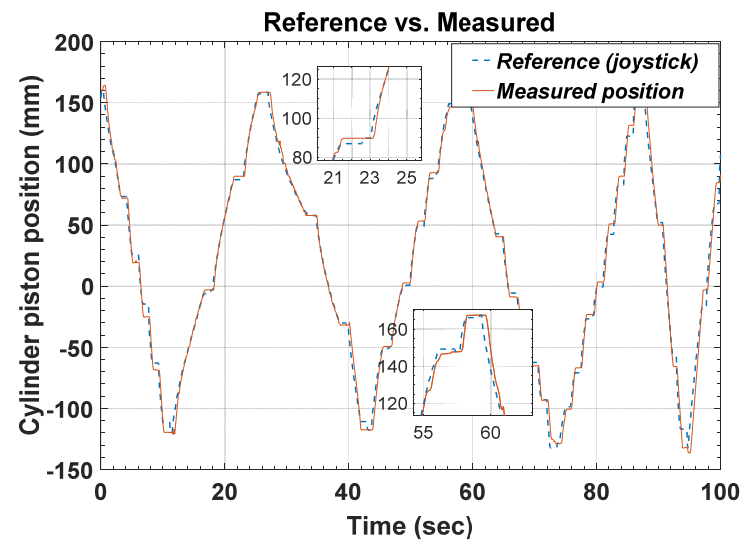

Fig.5. Reference tracking with joystick generated trajectory.

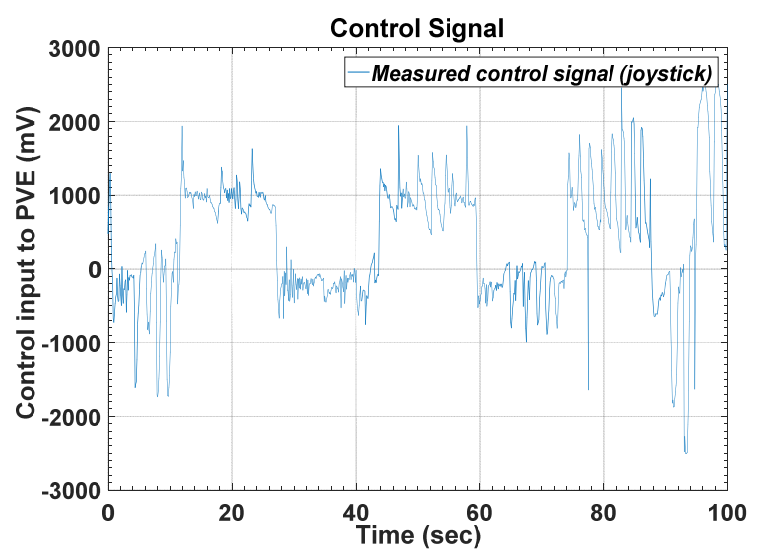

Fig. 6. Control signal to the PVE with joystick generated trajectory.

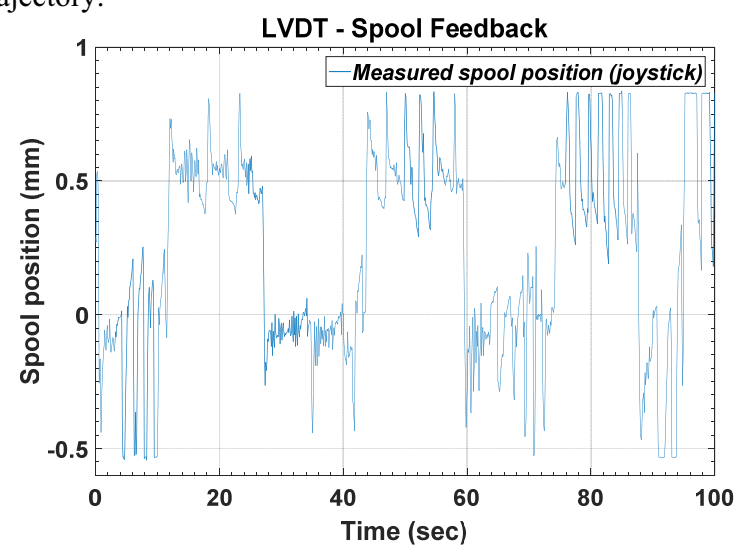

Fig.7. Spool position controller by the PVE with joystick reference trajectory.

The cascade control algorithm is programmed in the microcontroller MC012 with the help of Danfoss IDE PLUS 1 GUIDE [1]. It has standard capabilities similar to programming environments used in PLC (programmable logic controller) of other vendors. The resultant program is in the form of graphically connected blocks which comes from dedicated library and custom blocks. Custom blocks are described in structured text (STL) language. 


\subsection{Joystick generated reference signal}

The test-bench is equipped with single axis JS6000 Danfoss joystick. It can be programmed through CAN to filter the operator commands and outputs two PWM mutually exclusive sequences each corresponding to the position set point in each direction. When the sequence for the one direction is present the sequence for the other direction is not present. In this way the operator can send the commands to the microcontroller safely. The duty of the PWM signal is proportional to the desired position. When the stick of the joystick is moved by the operator the corresponding output signal begins to increase proportionally to the angle of the stick. So actually the operator commanded the desired velocity. As a result the generated reference signal can be described as

$$
r(t)=\left\{\begin{array}{l}
r_{1}(t), d_{\text {joystick }}>0 \\
r_{2}(t), d_{\text {joystick }}<0
\end{array}\right.
$$

where the $d_{\text {joystick }}$ is the displacement of the joystick introduced by the operator and $r_{1}, r_{2}$ are corresponding PWM sequences read by the microcontroller

$$
r_{k}(t)=\left\{\begin{array}{l}
0, t \bmod T_{j, p w m}<\gamma T_{j, p w m} \\
1, t \bmod T_{j, p w m}>\gamma T_{j, p w m}
\end{array},\right.
$$

and the PWM duty $\gamma$ is calculated by integrating the displacement of the joystick as

$$
\left\{\begin{array}{c}
\dot{\gamma}(t)=a\left|d_{\text {joystick }}(t)\right|, \gamma<1 \\
\dot{\gamma}(t)=0, \gamma=1
\end{array}\right.
$$

Fig. 5 shows the tracking performance when the joystick signal is used. The generated trajectory by the operator is a piecewise linear with quasi-periodic form spanning the whole stroke range of the servo cylinder. As can be observed from the figure the tracking error (a difference between desired trajectory and measured position) is very small (around 1 to $3 \mathrm{~mm}$ ). The largest value of the tracking error is during the transients when the operator desires to suddenly start or stop the steering. However, such value of the tracking error is acceptable for the steering applications of heavy mobile machinery.

Corresponding control action send from the microcontroller to the switching valve module (PVE) is shown in Fig. 6. As can be seen its amplitude stays in the prescribed limits of $\pm 5000 \mathrm{mV}$. This is an indication that the system can tolerate additional load disturbances or higher rates of reference change. However, the inherent dead-band nonlinearity present in the plant model limits the achievable tracking error by causing oscillatory behavior in steady state if the control action is further increased. Another thing evident from the control signal is the low levels of noises introduced by the position sensor.

Also the spool position used in the most internal control loop is presented in Fig. 7. It is measured in millimeter units. The maximal shift of the spool is around $3 \mathrm{~mm}$ in each direction. As can be seen, the EHSU system is highly sensitive to this signal with small displacement of the spool leading to immediate increase in the steering cylinder velocity.
Another interesting thing is that the spool position is slightly biased from the neutral position despite of the evident symmetry in the reference trajectory. This can be explained by some mechanical misfit in the unit which can be further studied so that it is reduced. However, the closed loop system functions normally even when such misfit is present.

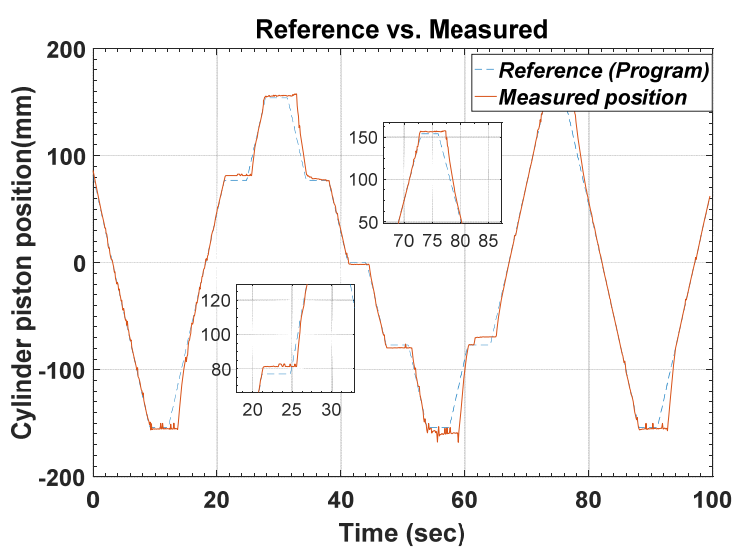

Fig.8. Reference tracking with pre-programmed trajectory.

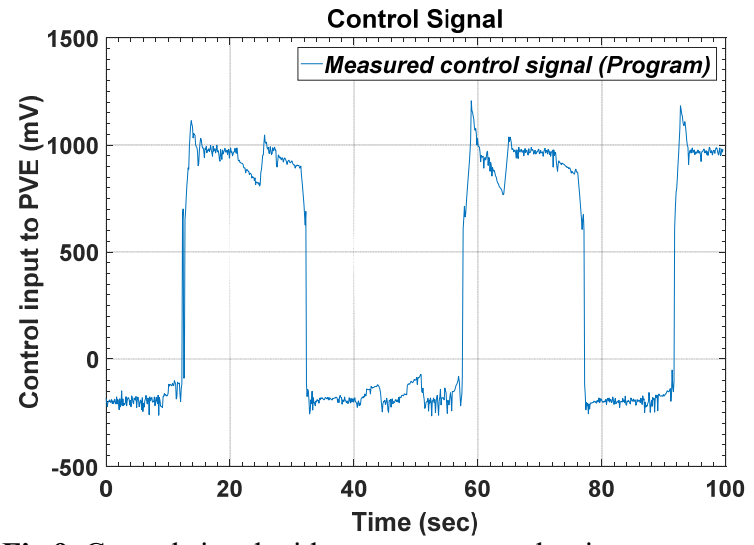

Fig.9. Control signal with pre-programmed trajectory.

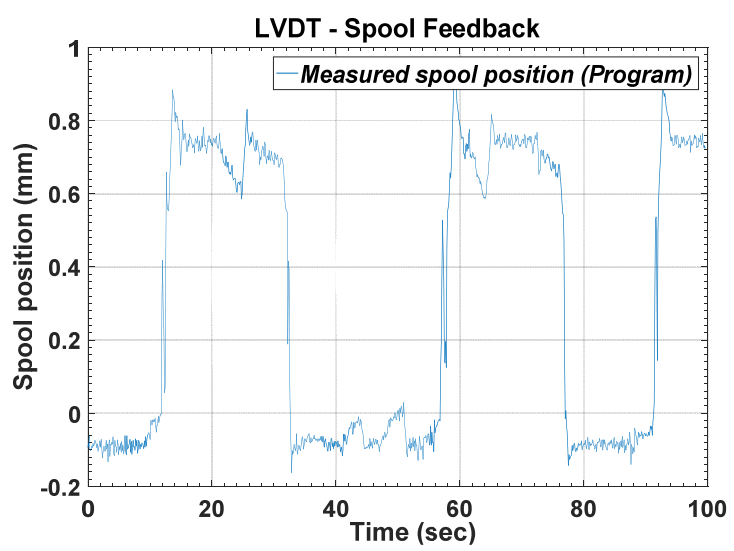

Fig. 10. Spool position controller by the PVE with preprogrammed trajectory.

\subsection{Pre-programmed reference signal}

In the second experiment the reference trajectory (Fig. 8) is pre-programmed in the microcontroller as a sequence of set point values evenly spaced in time. These values are generated with $\mathrm{MATLAB}^{\circledR}$ and recorded in 
CSV file which can be loaded in the microcontroller memory as one dimensional array [9]. As can be seen from Fig. 8, the results are similar to the case when a joystick generates the reference signal.

The corresponding control action sent from the microcontroller to the switching valve module (PVE) [10] is shown on Fig. 9. The spool position used in the most internal control loop is presented in Fig. 10.

\section{Conclusion}

The experimental results confirm closed-loop performance which is tested with two different reference trajectories typical for steering applications - joystick generated and pre-programmed. In both cases the tracking error is within the range of 1 to $3 \mathrm{~mm}$, the control signal stays within the prescribed limits of $\pm 5000 \mathrm{mV}$ and the spool position is within the limits of $\pm 3 \mathrm{~mm}$. The closed loop system has uniform performance for the whole stroke range of the cylinder $\pm 150 \mathrm{~mm}$. The system was tested with various loading pressures within the range between 2.5 to $7.5 \mathrm{MPa}$.

The developed and implemented laboratory bench for studying the load-sensing power steering system enables functional testing of modern electro-hydraulic steering devices designed for a wide range of mobile machinery.

The equipment of a variable displacement axial-piston pump with a pressure controller, as well as the incorporation of modern technical measuring instruments, enable the real-time record of a number of mechanical and hydraulic parameters. This in turn contributes to the study of the energy efficiency of the system with the inclusion of various control devices.

The present research was supported by funding contract № 181PR0020-02 of the Technical University of Sofia, Scientific and Research Sector (SRS) in the field of "Prospective Leaders". Also the authors are thankful to the conference organizing committee for their invitation.

\section{References}

1. Danfoss Inc., Steering, EHi steering valve, Technical Information, BC00000379en-US0202 (2018)

2. R. Isermann, Fahrdynamik-Regelung. Modellbildung, Fahrerassistenzsysteme, Mechatronik, ISBN 1038348-0109-7, (Friedr. Vieweg \& Sohn Verlag, Germany, 2006).

3. H. Murrenhoff, L. Eckstein, Fluidtechnik für mobile Anwendungen, ISBN 978-3-8440-0515-8, (ShakerVerlag, 2011)

4. K. Åström, T. Hagglund, PID Controllers: Theory, Design and Tunning, Second Edition, ISBN 1-55617516-7, United States of America (1995)

5. Rexroth Bosch Group, Axial Piston Variable Pump, A10VSO, serie 32, RE-A 92714 (2011)

6. J. Weber, Th. Neubert, E. Lautner, Arbeitsblätter zur Vorlesung: Steurungen, Softwareentwicklung und Sicherheit in mobile Anwendungen, Institut für Fluidtechnik (IFD), TU-Dresden (2016)
7. J. Weber, Arbeitsblätter zur Vorlesung: Mobilhydraulik, Institut für Fluidtechnik (IFD), TUDresden (2017)

8. A. Mitov, J. Kralev, Il. Angelov, Ts. Slavov, Identification and synthesis of linear-quadratic regulator for digital control of electrohydraulic steering system, $11^{\text {th }}$ International Fluid Power Conference, 1, 120-129, ISBN 978-3-95886-215-9, Germany (2018)

9. D. Gu, P. Petkov, M. Konstantinov, Robust Control Design with MATLAB ${ }^{\circledR}$. Springer-Verlag, London, second edition, ISBN 978-1-4471-4681-0 (2013)

10. A. Mitov, I. Angelov, J. Kralev, Embedded electrohydraulic controller with digital valve actuation for steering of heavy duty machines, $7^{\text {th }}$ Mediterranean Conference on Embedded Computing (MECO), 1, ISBN 978-1-5386-5684-6, Montenegro (2018) 